\title{
El texto literario en las clases de ELE: un recurso didáctico y motivador
}

\author{
Charo Nevado Fuentes \\ Universidad Pontificia de Salamanca \\ charonefu@gmail.com
}

Resumen: El presente artículo pretende poner de manifiesto la importancia que la literatura y en concreto el texto literario debe alcanzar en el campo educativo de la enseñanza de ELE. Abordaremos, desde una perspectiva histórica, las principales líneas de pensamiento que han tratado el tema de la enseñanza de la literatura en las clases de español como lengua extranjera, así como los diferentes tratamientos que el uso de la literatura ha tenido en el aula, pasando de ser un mero pretexto de presentación de contenidos a un recurso didáctico. También analizaremos las ventajas que proporcionan los textos literarios, ya que son muestras reales de lengua que complementan el proceso de enseñanza-aprendizaje. Por último, estableceremos los criterios de selección a la hora de elegir los textos literarios más adecuados para llevar al aula de español y presentaremos algunos ejemplos que sirvan como modelos de uso.

Palabras claveः Literatura, enseñanza-aprendizaje, recursos, programación, motivación

\begin{abstract}
The present article intends to evidence the importance that literature and in particular the literary text should reach in the Spanish as Foreign Language teaching educational system. We will tackle, from an historical point of view, the main currents of thought that have dealt with the subject of literature teaching in classes of Spanish as a Foreign Language. In addition we will address the different treatments that the use of literature has had in the classrooms, becoming an educational resource instead of a mere pretext for the presentation of contents. An analysis of the advantage of the literary texts will be performed, since they are real proofs of language that are complementary to the learning-teaching process. And last but not least, the selection criteria to choose the most appropriate literary texts for Spanish classes will be settled and we will submit some examples that serve as pattern of use.
\end{abstract}

Key words: Literature, teaching-learning, resources, curriculum, motivation 


\section{Introducción}

En el campo de la enseñanza del español como lengua extranjera, los docentes buscamos infinidad de recursos didácticos que nos ayuden en la transmisión de los contenidos y en la aplicación y puesta en práctica de los mismos. Muchas veces en esta búsqueda dejamos a un lado el componente literario en nuestras clases, ya que la palabra literatura implica dificultad y aburrimiento. El objetivo principal de este artículo es desmitificar esta idea y otorgar a los textos literarios la importancia que deben tener en el aula de ELE.

Es verdad que la literatura a lo largo de la historia ha estado presente en las diferentes corrientes metodológicas y educativas pero recibiendo tratamientos muy diferentes, que no siempre han perseguido un objetivo didáctico y motivador. Tradicionalmente, el texto literario desempeñó un papel fundamental en la enseñanza de lenguas, puesto que era considerado una obra maestra y un modelo a imitar en el proceso de aprendizaje de una segunda lengua. Posteriormente, y como consecuencia de la evolución metodológica, el texto literario ha dejado poco a poco de ser un mero pretexto para presentar los contenidos de aprendizaje, $y$ ha pasado a adquirir un papel relevante como recurso didáctico.

La literatura forma parte de la cultura y de la historia de un país, y por consiguiente tiene que formar parte del currículo de la enseñanza de lenguas, aspecto que queda reflejado en El Marco Común Europeo de Referencia para las Lenguas: aprendizaje, enseñanza y evaluación (MCER) ${ }^{1}$. No debemos confundir literatura, en su sentido más amplio, y el texto literario como muestra real de lengua ${ }^{2} . \mathrm{La}$ literatura entra a formar parte de las clases de español en sus cursos específicos de literatura como asignatura, y la utilización de los textos literarios en las clases de español son una herramienta esencial para el aprendizaje del léxico, de la gramática, la ortografía o de la cultura en sí misma. Por lo tanto, podemos considerar-

1 Dentro del MCER, en el capítulo 1: «El marco de referencia europeo en su contexto político y educativo, 1.2.: Los fines y los objetivos de la política lingüística del Consejo de Europa», se recoge lo siguiente: «Que el rico patrimonio de las distintas lenguas y culturas de Europa constituyen un recurso común muy valioso que hay que proteger y desarrollar, y que se hace necesario un importante esfuerzo educativo con el fin de que esta diversidad deje de ser un obstáculo para la comunicación y se convierta en una fuente de enriquecimiento y comprensión mutuos». Centro Virtual Cervantes, Marco común europeo de referencia para las lenguas: aprendizaje, enseñanza, evaluación, Instituto Cervantes, 2002-2008 [consulta: 12 de septiembre de 2014]. Disponible en la web: <http://cvc.cervantes.es/ensenanza/biblioteca_ele/marco/>.

2 Aunque el objetivo principal de este artículo es analizar las ventajas que ofrecen los textos literarios en el aula de español, es importante que los profesores de ELE establezcamos la diferencia entre «el uso literario de la lengua» $y$ «el uso comunicativo de la lengua»: La literatura, en la clase de español, puede ser abordada como un fin en sí misma, pero también como un recurso didáctico para la práctica de la lengua meta. Por lo tanto, esto no debe suponer una elección o una ruptura entre ambos términos. En relación a este tema podemos consultar las siguientes obras y artículos: Garrido, A. y Montesa, S. (1991). «La literatura en la enseñanza de español para extranjeros». En Actas de las III Jornadas internacionales del español como lengua extranjera. Las Navas del Marqués: 73-83; Prado, J. (2004). Didáctica de la lengua y la literatura para educar en el siglo XXI. Madrid: La Muralla. 
los como un elemento motivador orientado a potenciar los aspectos lingüísticoscomunicativos, y al desarrollo de las diversas destrezas y habilidades lingüísticas.

La elección de los textos literarios es una de las tareas fundamentales a tener en cuenta por parte de los docentes de español. Es importante que establezcamos unos criterios de selección para delimitar las características de los textos que vamos a utilizar, en correspondencia con los objetivos y contenidos lingüísticos, con los intereses y necesidades de los discentes y siempre adecuados a su nivel de aprendizaje.

Partiendo de estas reflexiones, los profesores de español tenemos que aprovechar las ventajas que conlleva la utilización del componente literario para llevarlo al aula e introducirlo en la programación como un recurso más dentro del aprendizaje.

\section{La literatura como parte del proceso educativo a través de la historia}

A lo largo de la historia de la metodología para la enseñanza de lenguas extranjeras, los textos literarios y, por extensión, la literatura, han tenido un papel fundamental, ya que siempre han formado parte del proceso de enseñanza-aprendizaje.

La lengua literaria ha recorrido toda la historia de la pedagogía, desde la Antigüedad grecolatina hasta el Diccionario de Autoridades ${ }^{3}$. Aunque la enseñanza de idiomas antes de la Edad Media ya tenía una larga trayectoria histórica ${ }^{4}$, será el latín el idioma que se consolide como lengua de comunicación entre diferentes pueblos; de este modo la enseñanza del latín se convierte en el modelo de enseñanza de lenguas.

Durante esta época la enseñanza del latín marca y consolida los hábitos de enseñanza y aprendizaje de lenguas. El aprendizaje se fundamenta en la gramática de Donato (siglo Iv) y, posteriormente en la gramática de Priscinano, Institutiones Grammaticae (siglo vi). En cuanto a la metodología, predomina la memorización de autores clásicos como Cicerón, Ovidio o Virgilio, entre otros. El objetivo

3 El Diccionario de Autoridades, publicado entre 1726 y 1739, se conoce como «de autoridades» por tener en los artículos citas de autores que ejemplifican o corroboran la definición dada. Entre los autores utilizados como autoridades de la lengua se incluyen los más ilustres representantes de la literatura castellana, sobre todo del Siglo de Oro, entre otros: Mateo Alemán, Santa Teresa de Jesús, Francisco de Quevedo, Luis de Góngora, Fray Luis de León, Antonio de Nebrija, Calderón de la Barca, Lope de Vega, Miguel de Cervantes.

4 Existen testimonios que reflejan que en el siglo iı a. de C. el aprendizaje de lenguas extranjeras era ya una realidad, $y$ aunque tal vez no fuera una enseñanza sistemática, el aprender una lengua con profesores nativos era considerado como algo conveniente y necesario, lo que ha llevado a ser motivo de interés, tanto su evolución a través del tiempo como la metodología. Cf., Kelly, L. (1969). 25 Centuries of language teaching. Rowley: Newbury House; Sánchez Pérez, A. (1997). Los métodos en la enseñanza de idiomas. Evolución bistórica y análisis didáctico. Madrid: Sgel; Titone, R. (1968). Teaching Foreign Languages: A Historical Sketch. Washington, D.C.: Georgetown University Press. 
principal es el análisis y la traducción de los textos literarios, para así llegar al conocimiento de la lengua y su cultura. El texto literario, como señala Hernández (1991: 9): «se concibe en esta época metodológica, como un pretexto para la transmisión de modelos». No persigue un fin comunicativo, por lo tanto muestra una falta de conexión con la realidad cotidiana y un alejamiento de la realidad comunicativa.

En el siglo xv y xvi, con la llegada del Renacimiento, se redescubren de nuevo los autores clásicos como Cicerón o Virgilio para el aprendizaje del latín culto. Los textos literarios de estos autores seguirán siendo el eje principal en la enseñanza de idiomas. Las lenguas vulgares, que fueron reemplazando poco a poco al latín como lenguas de comunicación, fueron transmitidas según el modelo de la escuela romana, es decir, se siguió el modelo de la enseñanza del latín durante la Edad Media.

En relación con la enseñanza del español como lengua extranjera ${ }^{5}$, nos tenemos que remontar al siglo xVI, cuando el español alcanzó su máximo esplendor llegando a ser el idioma más estudiado en Europa. La metodología utilizada sigue fielmente el modelo latino, y la literatura ocupa un lugar privilegiado tanto en la expansión de la cultura como en la enseñanza del castellano. El desarrollo literario que se produce en España en estos momentos traspasa fronteras; obras de nuestra literatura universal como La Celestina, El Lazarillo de Tormes, El Guzmán de Alfarache, El Amadís de Gaula o Don Quijote de la Mancha, entre otras, son traducidas a otros idiomas o editadas en español.

Junto con la aparición de gramáticas y diccionarios, la literatura tiene un gran peso en la enseñanza y difusión del español. Algunas de las obras más representativas de la literatura española de estos momentos incluyen, en anexos, explicaciones gramaticales, diccionarios o traducción de vocablos de la lengua meta a la lengua materna ${ }^{6}$. De este modo, ya en los inicios de la enseñanza del español como lengua extranjera, la literatura era un vehículo esencial para acercarse al castellano como segunda lengua.

En los siglos posteriores se producen nuevas aportaciones en lo referente a la enseñanza de lenguas extranjeras con la llegada de nuevos movimientos peda-

\footnotetext{
5 Más información sobre este tema puede consultarse en: Nevado Fuentes, C. (2004). «La difusión de la lengua española durante la Época Moderna: Algunos materiales didácticos». Revista Ciencias de la educación, número 200: 476-490.

6 Entre estas obras hay que destacar la edición veneciana de la obra de Fernando de Rojas La Celestina, publicada en 1556 y corregida por Alfonso de Ulloa. La obra contiene, en un anexo, una gramática y un vocabulario bilingüe en español y en italiano para facilitar la lectura a los que estudian la lengua castellana. Otra de estas obras es la primera parte de Don Quijote de la Mancha de Cervantes publicada en Francia, en París, por Cesar Oudin en 1614. En la traducción, al francés, se incluyen anotaciones al margen en castellano para facilitar al lector la lectura y conocer el contexto en el que se desarrolla la obra.
} 
gógicos y culturales como son el Realismo Pedagógico ${ }^{7}$ (xvir) y la Ilustración ${ }^{8}$ (xviII). Pero a pesar de estas aportaciones, el uso de la literatura en el campo de la educación no experimenta grandes cambios. Se seguirá fielmente el modelo latino para la enseñanza de una lengua extranjera, y en él se basará el primer método de enseñanza de idiomas, el método tradicional ${ }^{9}$, que surgirá a finales del siglo xviII.

El método tradicional o prusiano, también conocido como gramatical o de traducción, surgió a partir de 1845 y estuvo vigente hasta 1950. El objetivo lingüístico era capacitar al alumno para la lectura y el análisis de la literatura de la lengua meta. La enseñanza estaba basada en la traducción, la memorización y la imitación; así la literatura se convertía en el pretexto perfecto para la enseñanza de una segunda lengua.

A partir de 1950 se desarrolló el método oral o método estructural ${ }^{10}$. Los métodos estructurales o audiolinguales se fundamentan en la teoría lingüística del estructuralismo y la psicología conductista ${ }^{11}$, y suponen una ruptura con el modelo existente hasta ahora. Para estos métodos, el habla tiene prioridad sobre la lengua escrita, se defiende el aprendizaje de forma mecánica a través de la repetición e imitación de estructuras lingüísticas. Todo ello se traslada al ámbito de la didáctica de las lenguas extranjeras, que abandonan el uso del texto literario

7 En el siglo xvir surge el Realismo Pedagógico que supuso una notable transformación en el terreno educativo. Es un movimiento pedagógico crítico con la educación tradicional del Humanismo. El Humanismo valoraba más el texto o la cultura, en cambio, el Realismo Pedagógico trata de conseguir que lo aprendido sea utilizado. Destacan autores como Wolfgang Ratke (1571-1635), Juan Amós Comenio (1592-1670) o John Locke (1632-1704).

8 Es un movimiento cultural que surge en Europa a lo largo del siglo xviri. La Ilustración española supuso una crítica a la educación tradicional. Se inicia un proceso crítico a nuestra cultura, educación y mentalidad tradicionales. Más información sobre este tema puede consultarse en: Llopis, J. y Carrsco, M. V. (1983). Ilustración y Educación en la España del siglo XVIII. Valencia: Publicaciones de la Escuela Universitaria del Profesorado de EGB.

9 En el siglo xviır surge el primer método de enseñanza de lenguas extranjeras considerado una copia de los procedimientos utilizados para la enseñanza de las lenguas clásicas. En 1845, el profesor americano de lenguas clásicas, Sears, publicó un libro titulado El método ciceroniano o prusiano para la enseñanza de los elementos de la lengua latina, donde expuso los fundamentos del método tradicional que se había planteado a mediados del siglo XVIII pero que se desarrolló plenamente en el siglo xix. Se basaba fundamentalmente en la enseñanza del latín como lengua culta, pero posteriormente se desarrolló también para el aprendizaje de otras lenguas europeas. Cf., Kondo, C. M.; Fernández, C.; Higueras, M. (1997). Historia de la Metodología de Lenguas Extranjeras. Madrid: Fundación Antonio de Nebrija.

10 A mediados del siglo xx Charles Fries desarrolló el método oral o estructural. Fries dirigió el English Language Institute de la Universidad de Michigan. Aplicó los principios del estructuralismo a la enseñanza del inglés, lo que suponía un rechazo al método tradicional en favor de las estructuras y de la práctica a través de ejercicios orales de repetición de esas estructuras. Publicó en 1945 Teaching and Learning English as a Foreign Language.

11 Las teorías conductistas sostienen que el aprendizaje de una lengua extranjera se aprende mediante la imitación y la repetición, es decir, se imita y se repite lo que se oye a través del reforzamiento. 
como punto de partida para el aprendizaje de idiomas. Como apunta Hernández (1991: 12):

Abandonado el uso del texto literario como punto de partida para el aprendizaje de idiomas, el auge de los métodos audiolinguales y situacionales determinó un cambio radical en lo que al tratamiento de la lectura en la clase de lenguas extranjeras se refiere.

Como rechazo a los métodos anteriores, surgen a partir de los años 70 los métodos $\operatorname{cognitivos}^{12}$, que elaboran nuevas teorías sobre el aprendizaje de lenguas extranjeras. Se basan en la psicología cognitiva, que reivindica el papel creativo de la mente oponiéndose a la concepción conductista del aprendizaje. En la lingüística aplicada, el cambio propuesto por el cognitivismo se ve reflejado en los nuevos métodos que surgen en la enseñanza de lenguas ${ }^{13}$.

Con la llegada del método comunicativo, a principios de los años 70 , se produce un cambio significativo en la enseñanza de idiomas; la lengua se concibe como instrumento de comunicación, es decir, se promueve la comunicación en situaciones reales. No obstante, la literatura sigue sin estar presente en el proceso de enseñanza-aprendizaje, como señalan Collie y Slater (2002: 2): «el enfoque hacia los aspectos comunicativos del lenguaje del nuevo modelo provoca un marcado rechazo hacia la literatura».

La revalorización de la literatura como recurso didáctico para la enseñanza de idiomas cobra importancia en el último tercio del siglo xx. La enseñanza del inglés es pionera en este tema; así lingüistas como Widdowson ${ }^{14} y$ autores como Maley y Duff ${ }^{15}$ se plantean la incursión de los textos literarios como recurso para la enseñanza de segundas lenguas y no solamente como objeto de estudio literario.

En la enseñanza del español como lengua extranjera, los estudios son escasos. Es en los manuales o libros de texto donde los profesores de español podemos observar el tratamiento que la literatura ha tenido en la enseñanza de ELE.

12 En los métodos cognitivos la teoría del aprendizaje que subyace es el modelo innatista, que sostiene la existencia de una capacidad innata para desarrollar el lenguaje. $\mathrm{Y}$ al mismo tiempo se basan en el constructivismo defendido por Piaget, según el cual el aprendizaje de una lengua se concibe dentro del contexto de desarrollo intelectual del sujeto.

13 Los métodos cognitivos que surgieron a partir de este momento son muy variados, entre los que hay que destacar el Enfoque Natural, La Sugestopedia o el Enfoque Comunicativo.

14 Widdowson es uno de los principales defensores de la literatura en la enseñanza de lenguas extranjeras. Widdowson, H. G. (1975). Stylistics in the Teaching of Literature. Oxford: Oxford University Press.

15 Maley y Duff, en su obra, proponen diferentes técnicas didácticas para la explotación de textos poéticos. Parten del input poético para el desarrollo de las destrezas que forman parte del aprendizaje de una lengua. Cf., Maley, A. y Duff, A. (1989). The Inward ear Poetry in the Language Classroom. Cambridge: Cambridge University Press. 
En un primer momento, el texto literario está incorporado como apéndice introductorio o final de cada unidad sin perseguir ningún objetivo didáctico. Un estudio realizado por Ernesto Martín Peris en el año 2000, analiza el uso de textos literarios en seis manuales empleados en los años 80 y 90, y ahonda en esta idea:

El texto literario ha entrado de lleno en los manuales publicados en España [...] la ubicación general de los textos literarios es, casi de forma invariable, el final de las unidades. Esta tendencia a empujar hacia el final del proceso de aprendizaje, parece reflejar un cierto temor al texto literario: de tal modo que éste no sería visto como estímulo potenciador del aprendizaje, sino como gratificación reservada a quienes hayan logrado aprender. Complemento, por tanto, del aprendizaje, y no parte integrante del mismo. (Martín Peris, 2000: 123).

Posteriormente, encontramos ya manuales en los que poco a poco aparece algún texto literario que persigue unos objetivos concretos presentando actividades con una explotación lingüística. Por lo tanto, es importante que los docentes de español aprovechemos la riqueza que ofrecen los textos literarios, ya que son un excelente recurso a la hora de trabajar las habilidades lingüísticas en el aula de ELE.

\section{Ventajas del uso de la literatura en las clases de ELE}

Como hemos señalado anteriormente, el componente literario ha estado denostado durante tiempo de las clases de ELE al ser considerado un recurso demasiado complejo que obstaculizaba el proceso de enseñanza-aprendizaje. Pero estos argumentos, afortunadamente, van cambiando, y son muchos los autores que destacan la utilidad y eficacia del texto literario en la adquisición del español como lengua extranjera.

En los últimos años observamos una incorporación de la literatura en las aulas de lengua extranjera, ya sea como medio para el aprendizaje de aspectos léxico-gramaticales o como recurso didáctico para la producción de actividades. En este sentido, destacar los trabajos de los siguientes autores: (Byram y Fleming, 2001; Byram y Hammer, 2001).

A continuación vamos a reflexionar y a analizar algunas de las ventajas que nos proporcionan, a los docentes de ELE, la utilización del texto literario como recurso didáctico y parte del proceso de enseñanza-aprendizaje.

Una de las ventajas es el acercamiento cultural que las obras literarias proporcionan a los alumnos, puesto que son una muestra real que contextualizan el idioma en su contexto histórico, social y cultural. El texto literario permite interrelacionar lengua y cultura, ya que además de practicar la lengua, el alumno 
se ve inmerso en la cultura y la historia española. Sobre esta idea Sitman y Lerner (1994:231) afirman:

[... ] la enseñanza de idiomas extranjeros a través de la literatura en la lengua meta constituye, ante todo, un proceso interactivo de comunicación (entre el autor, el texto y el profesor como mediador) que puede contribuir al desarrollo de la interlengua y asimismo conducir al acercamiento cultural.

De esta forma, con el texto literario el alumno practica la lengua al mismo tiempo que se aproxima a su cultura. La literatura facilita al alumno el conocimiento de las tradiciones, costumbres y formas de vida de la lengua meta que está aprendiendo. Tal como señala Torres $(1998$ \$109):

[... enseñar una lengua extranjera es también enseñar la cultura de una o varias comunidades que la tienen como lengua materna y no hay nada a la vez tan próximo a una lengua y tan representativo de la cultura de una gente como su literatura.

Otra de las ventajas es que los textos literarios son un material real. Se presenta el uso del lenguaje en todas sus manifestaciones lingüísticas. En las muestras literarias encontramos diferentes contextos y situaciones que acercan a los alumnos a la vida cotidiana, por lo que contribuyen al desarrollo de la competencia comunicativa. Por lo tanto, el texto literario es un acto de habla cotidiano que presenta una gran variedad de recursos. Como observa Pedraza (1998: 61):

[...] la lengua real sólo tiene dos vías para presentarse ante el estudiante: 1) la comunicación espontánea con los nativos, que sólo es posible en un estadio avanzado del aprendizaje y en un lugar en que se hable el idioma que se pretende adquirir. 2) la lectura de textos literarios.

La dificultad lingüística, que en un principio puede conllevar la utilización de material auténtico, puede evitarse con la selección de un texto adecuado y con una buena explotación del material, a través de actividades diseñadas para conseguir un fin específico.

Otro de los puntos importantes es que son una herramienta fundamental para la práctica de las diferentes destrezas: mejoran la comprensión lectora; sirven como modelo para la práctica de la expresión escrita; también son un pretexto para la práctica de la expresión oral; y con el avance de las nuevas tecnologías se pueden utilizar materiales audiovisuales para la escucha o el visionado de muestras literarias, lo que nos lleva a la práctica de la comprensión auditiva.

Los textos literarios, al mismo tiempo, nos ayudan a transmitir una estructura gramatical o un elemento léxico. Por una parte, son un input comprensible para la 
presentación de contenidos lingüísticos. Y por otra parte, nos permiten presentar, practicar e incrementar el vocabulario.

Otro de los aspectos positivos es que al ser muestras reales de habla están siempre contextualizadas, lo que facilita la comprensión del alumno. El aprendizaje y la práctica de determinadas expresiones coloquiales o refranes resultan poco eficaces y motivadores si no las presentamos en un contexto real. Es por ello que no necesitamos recurrir a textos artificiales para encontrar situaciones que estén presentadas en contextos reales, pues en la literatura encontramos, por citar algunos ejemplos, diálogos, descripciones, narraciones, recomendaciones, etc. que favorecen el desarrollo de la competencia comunicativa.

Por último, los textos literarios son un excelente recurso para acercar al estudiante de español a la literatura española. Una vez que el estudiante toma contacto con el texto lograremos que se sienta atraído por la historia que se está contando, y tenga la necesidad de continuar su aprendizaje de español a través de la Literatura y no solo del texto literario.

En este sentido es importante la labor del profesor en la elección de textos literarios que involucren al alumno. Una vez que el lector entre en el mundo del texto y se sienta atraído por él, los aspectos lingüísticos que está estudiando pasan a un segundo plano y el desenlace de la obra pasa a ser el centro de atención.

\section{Criterios de selección. Explotación didáctica}

En relación a los criterios de selección, es fundamental la labor del profesor en la elección y selección de los textos literarios pues de ello dependerá su éxito en el aula de ELE.

Entendiendo como «textos literarios» o «materiales literarios» aquellos fragmentos de obras literarias que podemos utilizar en las clases de español con un fin didáctico para la consecución de unos objetivos previamente establecidos, los profesores tenemos que ser conscientes de que no todos los textos son válidos, por lo que es importante una correcta elección junto con una explotación didáctica adecuada de los mismos.

Una de las ventajas que nos proporciona la literatura es la gran variedad de géneros con los que podemos trabajar: la poesía, la novela, el teatro, el relato corto, las fábulas o los cuentos. Todos los géneros literarios tienen cabida en el aula de ELE, ya que cada uno de ellos presenta unas características específicas que podemos seleccionar $y$ adaptar perfectamente a los objetivos y contenidos marcados. Por lo tanto, el profesor de español tiene un abanico muy amplio para seleccionar el género literario y el texto que mejor se adecuen a las necesidades de aprendizaje. 
Para esta selección, el criterio más importante que se debe tener en cuenta es adecuar el texto literario al nivel de los discentes. Esto lo podremos utilizar en todos los niveles de aprendizaje, desde el nivel A1 al C2, pero para ello deben corresponderse con los contenidos lingüísticos de cada uno de los niveles. La lectura no tiene que suponer un reto o un fracaso en el alumno sino un recurso motivador que posibilite la consecución de los objetivos previstos.

Otro de los puntos a tener en cuenta es la explotación didáctica del texto literario. Todos los textos que llevemos al aula tienen que estar pensados con un fin concreto, es decir, unos objetivos que el alumno tiene que conocer de antemano. A partir de las actividades propuestas el alumno entrará en contacto con la lengua mediante la práctica y la producción.

De lo expuesto anteriormente, se deduce que los textos literarios deberían formar parte del currículo, algo que ya se contempla en el MCER ${ }^{16}$. Son un recurso más que los docentes de ELE tienen que tener en cuenta, ya que contribuyen al desarrollo de la competencia lingüística y al mismo tiempo potencian la imaginación y la creatividad.

Además de estos criterios, es esencial, por una parte, que los textos sean motivadores y significativos. Un texto que presente una historia interesante y divertida hará que los alumnos se involucren de forma positiva en el proceso de aprendizaje y por consiguiente, consigan los objetivos propuestos. Por otra parte, los gustos e intereses de los alumnos tienen que formar parte, en la medida de lo posible, de esta selección. Si un texto suscita el interés de los alumnos, la motivación está garantizada.

Por último, es conveniente que los textos que elijamos no sean demasiado extensos. El objetivo no es leer parte de una obra literaria, sino escoger fragmentos claros y contextualizados que sirvan como pretexto. Nuestro objetivo principal es conseguir que sean un recurso motivador en el aula de ELE para la práctica del español, y que no supongan un problema que dificulte el proceso de aprendizaje. La extensión vendrá marcada por el nivel de aprendizaje de los discentes.

Las actividades que acompañan a los fragmentos literarios son la parte esencial del proceso de enseñanza-aprendizaje. Sin esta explotación didáctica, el uso del material literario en las clases de español no tendría ningún sentido.

16 Dentro del MCER, en el capítulo 4: El Uso de la lengua y el usuario o alumno, en el punto 4.3.: Tareas y propósitos comunicativos hay un apartado que hace referencia a los usos estéticos de la lengua recogiendo algunos ejemplos de actividades. En este punto además se señala: «Los usos imaginativos y artísticos de la lengua son importantes tanto en el campo educativo como en sí mismos. Las actividades estéticas pueden ser de expresión, de comprensión, interactivas o de mediación [...]. Los estudios literarios cumplen muchos más fines educativos, intelectuales, morales, emocionales, lingüísticos y culturales que los puramente estéticos». Centro Virtual Cervantes, Marco común europeo de referencia para las lenguas: aprendizaje, enseñanza, evaluación, Instituto Cervantes, 2002-2008 [consulta: 12 de septiembre de 2014]. Disponible en la web: <http://cvc.cervantes.es/ ensenanza/biblioteca_ele/marco/>. 
En cuanto a las actividades propuestas, estas pueden estar diseñadas para la presentación de contenidos de forma deductiva o inductiva, para la práctica de los mismos, para la producción de determinadas estructuras o para un repaso final. Podemos practicar la gramática, el vocabulario, la ortografía, la fonética, y por supuesto tienen cabida todas las destrezas, como hemos señalado anteriormente.

La creación de actividades desde un punto de vista didáctico puede ser abordada desde diferentes planteamientos y en este caso expondremos tres usos pedagógicos: como elemento gramatical o léxico, como estímulo y como recurso. Por supuesto, no son los únicos ni se excluyen unos a otros, ya que todos se complementan, sirven ante todo para dejar patente el gran potencial de los textos literarios y para que los docentes de ELE se acerquen al texto literario para empezar a crear sus propias actividades:

1. Como herramienta para activar la competencia gramatical o léxica. La forma más tradicional es presentarlos con el fin de realizar ejercicios de gramática y vocabulario (completar espacios en blanco, ejercicios de relacionar, sinónimos, antónimos, etc.). Es el uso del texto literario centrado en el contenido lingüístico, es decir, el uso del texto nos ayuda a adquirir o practicar elementos particulares del léxico o de estructuras gramaticales.

Este tipo de ejercicios deberán ir siempre acompañados de actividades complementarias (pre-lectura o pos-lectura) que fomenten la producción y la interacción del alumno. Si partimos del texto literario como presentación o práctica de contenidos, tenemos que conseguir que éste se convierta en un recurso didáctico, a través de una batería de actividades que potencien el interés y la implicación de los alumnos.

Los dos ejemplos que mostramos a continuación no se presentarán de forma aislada, sino dentro de una secuencia de actividades orientadas a poner en práctica los contenidos adquiridos y acercar al alumno a la literatura (leer, buscar información u ordenar la biografía de los autores; descripciones de los personajes principales de Don Quijote; escribir un poema de cómo sería su mundo al revés...). 
Ejemplo 1: Completa con las preposiciones adecuadas la aventura de Don Quijote con los molinos de viento.

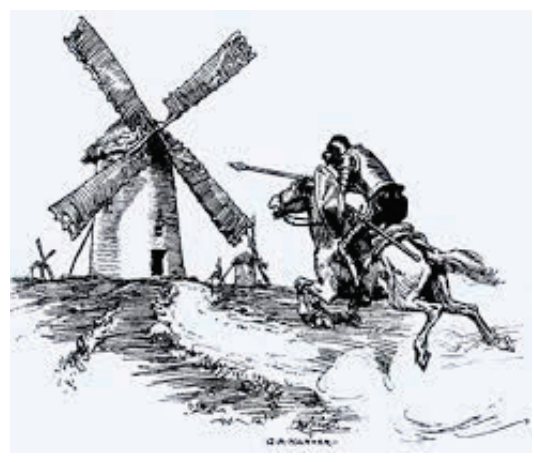

En esto descubrieron treinta o cuarenta molinos viento que hay aquel campo,

y así como Don Quijote los vio, dijo $\mathrm{su}$ escudero: la ventura va guiando nuestras cosas mejor lo que acertáramos desear; porque ves allí, amigo Sancho Panza, donde se descubren treinta o poco más desaforados gigantes quien pienso hacer batalla, $y$ quitarles todos las vidas, cuyos despojos comenzaremos enriquecer:

que esta es buena guerra, y es gran servicio de Dios quitar tan mala simiente de sobre la faz de la tierra. ¿Qué gigantes? dijo Sancho Panza.

Miguel de Cervantes, Don Quijote de La Mancha.

Ejemplo 2: Escribe el antónimo de los adjetivos que aparecen en el texto.
Érase una vez un lobito bueno al que maltrataban todos los corderos. Había también una bruja hermosa un príncipe malo $y$ un pirata honrado. Todas estas cosas había una vez cuando yo soñaba un mundo al revés.

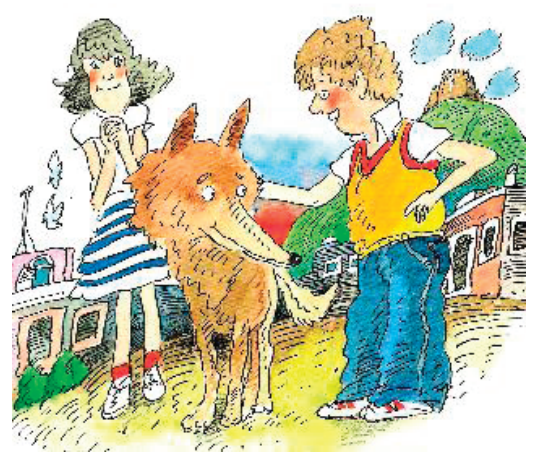

José Agustín Goytisolo, Los poemas son mi orgullo: antología poética.

2. Utilizar los textos literarios como estímulo. Un estímulo orientado a otra tarea u otro tipo de discusión. El texto literario fomenta la reflexión y la creación. Los estudiantes a través de la lectura tienen que adivinar de qué trata la lectura y posteriormente elaborar sus propias historias. El objetivo principal no es la adquisición del léxico pero sí está presente como objetivo secundario. 


\section{Ejemplo 3:}

- Lee el siguiente texto y después por parejas adivinad de qué animal se trata.

- Haz una descripción de un animal siguiendo la descripción de Platero (descripción física, psicológica, qué hace, qué le gusta).

- Lee la descripción que has escrito a tus compañeros para ver si adivinan tu animal.

Platero es pequeño, peludo, suave; tan blando por fuera, que se diría todo de algodón, que no lleva huesos. Sólo los espejos de azabache de sus ojos son duros cual dos escarabajos de cristal negro.

Lo dejo suelto, y se va al prado, y acaricia tibiamente con su hocico, rozándolas apenas, las florecillas rosas, celestes, gualdas... Lo llamo dulcemente: «Platero?», y viene a mí con un trotecillo alegre que parece que se ríe, en no sé qué cascabeleo ideal...

Come cuanto le doy. Le gustan las naranjas mandarinas, las uvas moscateles, todas de ámbar, los higos morados, con su cristalina gotita de miel...

Es tierno y mimoso igual que un niño, que una niña...; pero fuerte y seco por dentro, como de piedra. Cuando paso sobre él, los domingos, por las últimas callejas del pueblo, los hombres del campo, vestidos de limpio y despaciosos, se quedan mirándolo: -Tien'asero...

Tiene acero. Acero y plata de luna, al mismo tiempo.

Platero y yo, Juan Ramón Jiménez.

3. El uso del texto literario como recurso. Los estudiantes interactúan con un texto que les proporciona algún tipo de información. Es decir, el texto es contenido, es input. En este sentido es un recurso o fuente de información. En el ejemplo que proponemos a continuación partimos de un input gramatical que en este caso son los imperativos para la práctica de las órdenes. La actividad es un desencadenante para activar ese contenido lingüístico.

\section{Ejemplo 4:}

- Lee la siguiente lectura.

- ¿De qué trata el texto?

- ¿Qué verbos se utilizan?

- ¿Cuál es el uso de los verbos que aparecen?

- Escribe con tu compañero las órdenes que os daban vuestros padres cuando eráis pequeños. 
No cojas la cuchara con la mano izquierda.

No pongas los codos en la mesa.

Dobla bien la servilleta.

Eso, para empezar.

Extraiga la raíz cuadrada de tres mil trescientos trece.

¿Dónde está Tanganika? ¿Qué año nació Cervantes?

Le pondré un cero en conducta si habla con su compañero.

Eso, para seguir.

$¿$ Le parece a $\mathrm{Ud}$. correcto que un ingeniero haga versos?

La cultura es un adorno y el negocio es el negocio.

Si sigues con esa chica te cerraremos las puertas.

Eso, para vivir.

No seas tan loco. Sé educado. Sé correcto.

No bebas. No fumes. No tosas. No respires.

¡Ay sí, no respires! Dar el no a todos los «no»

$Y$ descansar: Morir.

Gabriel Celaya, Autobiografía.

Es importante recalcar que los textos elegidos para su explotación debemos aprovecharlos exhaustivamente, procurando realizar el mayor número de actividades. Podemos utilizar el texto con un objetivo meramente lingüístico, como hemos visto en los dos primeros ejemplos, pero siempre iremos más allá para que tengan cabida al mismo tiempo otras habilidades comunicativas (hablar, escribir, leer, escuchar). En el texto de don Quijote, podemos complementar la actividad con el visionado de Los molinos de viento. Y en el caso del Lobito bueno, con la audición de Paco Ibáñez. En definitiva, que los estudiantes a través de diferentes actividades interactúen, se impliquen y trabajen la adquisición de la lengua partiendo de un modelo literario.

Todas las obras de la literatura española e hispanoamericana ${ }^{17}$ pueden formar parte de nuestra selección, y son múltiples los fragmentos que podemos extraer de ellas para llevar al aula y complementar el proceso de adquisición del español como lengua extranjera.

Entre ellas podemos citar algunos ejemplos: El cuento de la Lechera de don Juan Manuel, incluido en El Conde Lucanor, para la práctica de los pasados. Misericordia de Benito Pérez Galdós, para la práctica de la descripción. Como agua

17 No debemos olvidarnos de la literatura hispanoamericana, ya que forma parte de nuestra riqueza lingüística y cultural. 
para chocolate de Laura Esquivel, para la práctica de las recetas de cocina. Sin noticias de Gurb de Eduardo Mendoza, para la práctica de la escritura creativa. El móvil de Juan José Millás, para la práctica de la probabilidad. La tía Tula de Unamuno, para la práctica del diálogo.

En último lugar, no debemos olvidar que existen adaptaciones cinematográficas de numerosas obras literarias ( $\mathrm{La}$ Colmena de Camilo José Cela, La Casa de Bernarda Alba de Federico García Lorca, La tía Tula de Miguel de Unamuno, El maestro de Esgrima de Arturo Pérez Reverte, Fortunata y Jacinta de Benito Pérez Galdós, La casa de los espíritus de Isabel Allende, etc.), y canciones de los poemas más importantes de nuestra literatura (poemas de Antonio Machado o Miguel Hernández cantados por Joan Manuel Serrat, El lobito bueno cantado por Paco Ibáñez, Poemas de Pablo Neruda cantados por Mercedes Sosa, etc.). Esto nos permite a los profesores de español contar con un material complementario que hace nuestras clases más variadas y dinámicas.

\section{A modo de conclusión}

A lo largo de estas páginas hemos podido observar la importancia que la literatura tiene en la enseñanza del español como lengua extranjera. Las ventajas que los textos literarios aportan a la enseñanza de lenguas son innumerables, ya que son un valioso recurso didáctico que potencia la motivación.

Utilizar los materiales literarios en el aula de español con fines didácticos permite fomentar los aspectos socioculturales y al mismo tiempo desarrolla y favorece la competencia pragmática y comunicativa.

Aunque hemos avanzado mucho en este campo, todavía queda camino por recorrer. Los profesores de español tenemos que adentrarnos en nuestra literatura para descubrir aquellos textos literarios que se adecuan a los objetivos y contenidos de nuestras clases con el fin de incluirlos en el proceso de enseñanzaaprendizaje.

Para los profesores, la literatura es una herramienta fundamental con la que contamos, ya que nos permite utilizar, adaptar y crear actividades de grandes obras literarias. La lectura genera placer y aplicándola a la enseñanza de lenguas potencia el aprendizaje. Si además conseguimos que nuestros alumnos disfruten de la lectura, estaremos consiguiendo que valoren la obra literaria y sientan la necesidad de adentrarse en la literatura de habla hispana. 


\section{Referencias bibliográficas}

Byram, M. y Fleming, M. (2001). Perspectivas interculturales en el aprendizaje de idiomas. Enfoques a través del teatro y la etnografía. Madrid: CUP.

Byram, M. y Hammer, M. (2001). Perspectivas interculturales en la enseñanza de lenguas extranjeras. Ideas a partir del teatro y la etnografía. Madrid: CUP.

Celaya, G. (2004). Poesías completas. Madridः Visor libros.

Centro Virtual Cervantes. Marco común europeo de referencia para las lenguas: aprendizaje, enseñanza, evaluación, Instituto Cervantes, 2002-2008 [consulta: 12 de septiembre de 2014]. Disponible en la web: <http://cvc. cervantes.es/ensenanza/biblioteca_ele/marco/>.

Cervantes Sanedra, M. (1979). Don Quijote de la Mancha. Madrid: EspasaCalpe.

Collie, J. y Slater, S. (2002). Literature in the Language Classroom: A resource book of ideas and activities. Cambridge: Cambridge University Press.

Garrido, A. y Montesa, S. (1991)* «La literatura en la enseñanza de español para extranjeros». En Actas de las III Jornadas internacionales del español como lengua extranjera. Las Navas del Marqués: 73-83.

Goytisolo, J.A. (2003). Antología poética. Barcelona: Lumen.

Hernández Blasco, M. J. (1991). «Del pretexto al texto. La lectura en la enseñanza-aprendizaje de idiomas y su tratamiento en español como lengua extranjera». Cable, Revista de didáctica del español como lengua extranjera, $\mathrm{n}^{\circ} 7$, Madrid, ed., Equipo Cable: 9-13.

Higueras, M. (1997). Historia de la Metodología de Lenguas Extranjeras. Madrid: Fundación Antonio de Nebrija.

Jiménez, J.R. (2005). Platero y yo. Madrid: Cátedra.

Kelly, L. (1969). 25 Centuries of language teaching. Rowleyः Newbury House.

Kondo, C. M.; Fernández, C.; Higueras, M (1997). Historia de la Metodología de Lenguas Extranjeras. Madrid: Fundación Antonio de Nebrija.

Llopis, J. y Carrsco, M. V. (1983). Ilustración y Educación en la España del siglo XVIII. Valencia: Publicaciones de la Escuela Universitaria del Profesorado de EGB.

Maley, A. y Duff, A. (1989). The Inward ear Poetry in the Language Classroom. Cambridge: Cambridge University Press.

Martín Peris, E. (2000). «Textos literarios y manuales de enseñanza de español como lengua extranjera», Lenguaje y textos, $\mathrm{n}^{\circ} 16: 101-129$.

Naranjo Pinta, M. (1999). La poesía como instrumento didáctico en el aula de español como lengua extranjera. Madrid: Edinumen. 
Nevado Fuentes, C. (2004). «La difusión de la lengua española durante la Época Moderna: Algunos materiales didácticos», Revista Ciencias de la educación, número 200: 476-490.

Pedraza Jiménez, F. B. (1998). «La literatura en la clase de español para extranjeros». En Celis, A. y Heredia, J. R. (Coords.) Actas del VII Congreso Internacional de ASELE. Lengua y cultura en la enseñanza del español a extranjeros, Cuenca, Universidad de Castilla La Mancha: 59-66.

Pozuelo, J. M. (1983). La lengua literaria. Málaga: Librería Ágora.

Prado, J. (2004). Didáctica de la lengua y la literatura para educar en el siglo XXI. Madrid: La Muralla.

Richards, J. C. y Rodgers, T. S. (2001). Approaches and Methods in Language Teaching. Cambridge: Cambridge University Press.

Sánchez Pérez, A. (1997). Los métodos en la enseñanza de idiomas. Evolución bistórica y análisis didáctico. Madrid: Sgel.

Sitman, R. y Lerner, I. (1994). «Literatura hispanoamericana: herramienta de acercamiento cultural en la enseñanza del español como lengua extrajera». En J. Sánchez Lobato e I. Santos Gargallo, (Eds.) Actas del IV Congreso Internacional de ASELE. Problemas y métodos en la enseñanza del español como lengua extranjera. Madrid, Sgel: 227-233.

Titone, R. (1968). Teaching Foreign Languages: A Historical Sketch. Washington, D.C.: Georgetown University Press.

Torres, C. (1998). «El texto literario: subsidio mediato para la enseñanza del español como lengua extranjera». En Sánchez Lobato, J. y Santos Gargallo, I. (Eds.) Actas del VI Seminario de dificultades especificas para la enseñanza del español a lusohablantes. Consejería de Educación y Ciencia de la Embajada de España en Brasil: 104-110.

Widdowson, H. G. (1975). Stylistics in the Teaching of Literature. Oxford: Oxford University Press. 